\title{
Uma Plataforma para Apoio à Segurança em Campus Inteligente
}

\author{
Silvino Medeiros, Ícaro França, Eiji Adachi, José Alex Lima, \\ Frederico Lopes, Everton Cavalcante, Nélio Cacho
}

\author{
Universidade Federal do Rio Grande do Norte (UFRN) \\ Natal-RN, Brasil \\ \{silvinogustavo, icazevedo10, j.alex.medeiros\}@gmail.com, \\ \{eijiadachi, fred\}@imd.ufrn.br, \{everton, neliocacho\}@dimap.ufrn.br
}

\begin{abstract}
Resumo. Esse artigo versa sobre as necessidades encontradas no contexto de segurança na Universidade Federal do Rio Grande do Norte (UFRN), em Natal$R N$, e propõe o SIGOc - Sistema Integrado de Gestão de Ocorrências, uma plataforma que objetiva otimizar o modo atual de operação para o gerenciamento de ocorrências nos campi da UFRN. O SIGOc consiste em dois aplicativos para dispositivos móveis e um sistema Web, permitindo que a comunidade universitária reporte ocorrências e, ao mesmo tempo, apoiando o trabalho das equipes de segurança do campus para um rápido atendimento de ocorrências. Além disso, a solução proposta visa endereçar os desafios encontrados nesse contexto em três frentes principais, a saber, gerencial, informacional e comunicacional.
\end{abstract}

\begin{abstract}
This paper deals with needs related to safety in the Federal University of Rio Grande do Norte (UFRN), in Natal, and proposes SIGOc-Integrated System for Occurrence Management, a platform that aims to optimize the current operation mode to manage occurrences in the UFRN campuses. SIGOc consists of two mobile applications and a Web system, enabling the university community to report occurrences while supporting the work of campus safety staff for fast occurrence handling. Moreover, the proposed solution seeks to address challenges found in this context on three main fronts, namely managerial, informational, and communicational.
\end{abstract}

\section{Introdução}

Na visão de Barrionuevo et al. (2012), uma cidade inteligente utiliza todos os recursos tecnológicos disponíveis de maneira coordenada e inteligente para desenvolver centros urbanos que são integrados, habitáveis e sustentáveis. Nesse mesmo contexto, Lacinák et al. (2017) afirmam que toda cidade inteligente precisa ser uma cidade segura e que centros urbanos com essa característica devem possuir, dentre outros mecanismos, sistemas inteligentes para monitoramento, procura, detecção e identificação de crimes e eventos que ameacem a segurança pública.

Nos últimos anos, houve um aumento significativo nos índices de criminalidade nas grandes cidades brasileiras. Segundo dados do Atlas da Violência ${ }^{1}$, a taxa de homicídios a cada 100 mil habitantes no Brasil foi de 26,1 para 28,9 no período de 2005 a

${ }^{1}$ http://ipea.gov.br/atlasviolencia/ 
2015, taxa aproximadamente quatro vezes maior do que a média do planeta. Nesse mesmo período, essa taxa passou de 13,5 para 44,9 homicídios no Estado do Rio Grande do Norte, representando uma variação de aproximadamente $231,99 \%$, alcançando a maior variação entre todas as unidades federativas do País.

Com esse aumento da criminalidade e o crescimento da população acadêmica nos campi universitários brasileiros, os desafios de segurança enfrentados por uma cidade refletem-se também dentro desses espaços. Afinal, os campi universitários estão localizados dentro das cidades e tipicamente não há barreiras físicas que delimitem ou impeçam o livre acesso e trânsito de pessoas pelas dependências desses campi. A despeito das semelhanças entre cidades e campi universitários, a questão de segurança desses espaços guarda algumas particularidades. Enquanto nas cidades as responsabilidades da segurança pública ficam bem definidas entre Polícia Civil (policiamento investigativo) e Polícia Militar (policiamento ostensivo), tais responsabilidades não são tão claras assim nas dependências dos campi universitários. Por um lado, alguns defendem que, por ser papel da Polícia Militar (definido no Art. 144 da Constituição Federal do Brasil) a preservação da ordem pública, caberia também a ela atuar na segurança das universidades públicas por estas serem bens públicos. Por outro lado, há quem defenda que as universidades federais são autarquias com personalidade jurídica própria e, portanto, não caberia à Polícia Militar atuar em suas dependências. É fato que, na maioria dos campi de universidades federais, a atuação da Polícia Militar é assunto polêmico e que tais instituições costumam possuir órgãos próprios para a garantia da segurança em suas dependências.

No contexto da Universidade Federal do Rio Grande do Norte (UFRN), no Estado do Rio Grande do Norte, a responsabilidade da segurança universitária é da Divisão de Segurança Patrimonial (DSP), a qual agrega servidores públicos e funcionários terceirizados. É de responsabilidade dos agentes da DSP o atendimento de ocorrências diversas, desde ocorrências mais brandas como a detecção de veículos abertos ou acidentes a ocorrências emergenciais como assaltos e arrombamentos de veículos e prédios. Em um estudo realizado com a DSP, foram identificado alguns desafios que prejudicam os processos de registros de ocorrências de segurança na Universidade. Em particular, esses desafios podem ser caracterizados em três tipos: (i) desafios comunicacionais, que dizem respeito à comunicação tanto entre a comunidade universitária e a DSP quanto entre os supervisores da DSP e seus vigilantes; (ii) desafios gerenciais, que se relacionam aos processos de monitoramento e alocação de recursos, e; (iii) desafios informacionais, relacionados à maneira como os dados dos processos de gerenciamento são armazenados, analisados, visualizados e compartilhados.

Até o momento, tem-se conhecimento de poucas soluções endereçando do ponto de vista tecnológico a questão da segurança em campi universitários. Na direção de melhorar a segurança dentro de seus campi, a Universidade de São Paulo (USP) desenvolveu uma solução que consiste em um ponto de entrada para registrar ocorrências de segurança diretamente para o Departamento de Segurança da Universidade [Ferreira et al. 2017]. Outras propostas existentes são o AppArmor ${ }^{2}$ e o LiveSafe ${ }^{3}$, que contam respectivamente com a definição de perímetros virtuais com base na localização de dispositivos (geofencing) e chamadas de emergência através de um aplicativo móvel. Ainda assim, nenhum

${ }^{2} \mathrm{https}: / /$ www.apparmor.com/

${ }^{3}$ https://www.livesafemobile.com/solutions/ 
dessas propostas aborda os desafios relacionados à segurança em campi anteriormente mencionados.

Nesse contexto, este artigo apresenta o SIGOc - Sistema Integrado de Gestão de Ocorrências, uma plataforma desenvolvida no contexto do Projeto Smart Metropolis ${ }^{4}$ do Instituto Metrópole Digital (IMD) da UFRN com o intuito de apoiar as atividades de gerenciamento da segurança universitária realizadas pela DSP-UFRN, além de facilitar a comunicação entre ela e a comunidade universitária. A solução consiste no desenvolvimento e utilização dos seguintes sistemas:

(i) um aplicativo para dispositivos móveis para que as pessoas possuem algum vínculo com a universidade (discentes, docentes e servidores técnicoadministrativos) possam registrar ocorrências de segurança;

(ii) um aplicativo móvel para a equipe de segurança da DSP, para que as ocorrências possam ser atribuídas de maneira mais confiável e rápida, e;

(iii) um sistema Web de gerenciamento das ocorrências, em que funcionários da DSP poderão despachar equipes de segurança para o atendimento de ocorrências, receber chamados, gerar relatórios e acompanhar o posicionamento da segurança universitária em tempo real.

O restante deste artigo está estruturado da seguinte forma. A Seção 2 apresenta uma visão geral dos desafios enfrentados pela DSP no gerenciamento da segurança pública na UFRN. O SIGOc, seus elementos e as funcionalidades de cada um são descritos na Seção 3. A Seção 4 analisa o problema comparativamente, mostrando como cada componente contribui para a otimização do modo atual de operação para o gerenciamento de ocorrências nos campi da UFRN. A Seção 5 trata acerca de alguns trabalhos a serem realizados futuramente com base na solução proposta. Por último, a Seção 6 traz algumas considerações finais.

\section{Desafios de Segurança num Campus Universitário}

No contexto da UFRN, foi possível observar que, ao longo dos anos, a obsolescência dos métodos de gerenciamento das atividades de segurança universitária acarretou na imprecisão dos dados coletados sobre as ocorrências de segurança em seus campi. $\mathrm{O}$ registro de ocorrências era feito pelos vigilantes manualmente em um caderno de ocorrências na DSP ao fim do expediente de cada vigilante. Dessa forma, não havia garantias de que todas as ocorrências atendidas pelos vigilantes ao longo do dia eram de fato registradas nem se estas eram feitas com o nível de detalhes que se esperaria. Esse fato inclusive dificultou a realização de uma avaliação situacional da segurança nos campi da UFRN ou da qualidade do serviço prestado pela DSP, uma vez que, com poucos registros oficiais, não era possível definir indicadores precisos sobre ocorrências registradas e atendidas ao longo dos anos.

Outra limitação observada no contexto da segurança da UFRN foi o fato de comunicação entre os supervisores da DSP e os vigilantes em ronda pelos campi ser toda realizada via rádio. Informações sobre a localização dos vigilantes, para saber qual deles estava mais próximo a uma determinada ocorrência, eram passadas entre todos os vigilantes em serviço e os supervisores. De forma similar, detalhes das ocorrências como

\footnotetext{
${ }^{4} \mathrm{http}: / /$ smartmetropolis.imd.ufrn.br/
} 
seu tipo, sua localização, características dos indivíduos e/ou ferramentas envolvidas e autor(a) do cadastro da ocorrência também eram passadas via rádio. $\mathrm{O}$ principal problema nesta forma de comunicação era o alcance do rádio, o qual nem sempre funcionava corretamente: o campi central da UFRN, localizado em Natal, é de grandes dimensões (123 hectares), enquanto nos campi localizados no interior do Estado do Rio Grande do Norte sequer havia a possibilidade de se usar o rádio. Havia ainda o problema da falta de registro das informações trocadas via rádio, novamente contribuindo para a perda de dados e dificultando a realização análises quanto às ocorrências registradas e atendidas e quanto à qualidade do atendimento das ocorrências. Adicionalmente, observaram-se atrasos no atendimento das ocorrências devido à natureza conversacional do processo de despacho de vigilantes e também por eventuais indisponibilidades do canal de comunicação.

Observou-se ainda que havia apenas um canal de comunicação através do qual a comunidade universitária podia reportar ocorrências de segurança observadas nos campi da UFRN, a saber, uma linha telefônica na central da DSP. Indivíduos da comunidade acadêmica precisavam telefonar para a DSP para reportar e descrever uma ocorrência de segurança e, por sua vez, os supervisores da DSP anotavam estas informações e as repassavam aos vigilantes para que estes fossem atender a ocorrência. $\mathrm{O}$ principal problema desse canal de comunicação era o seu desconhecimento por grande parte da comunidade universitária, além da falta de registro oficial das informações reportadas pela comunidade universitária aos supervisores da DSP. Ainda que existisse registro de algumas informações, a DSP não possuía ferramentas de análise de dados, ou seja, havia dificuldade em correlacionar informações sobre ocorrências anteriores e a visualização desses dados consistiam em formatos que empobrecem o entendimento deles, tais como simples planilhas e documentos de texto.

Analisando os desafios encontrados no contexto de gerenciamento de segurança na UFRN, foi feita uma caracterização dos desafios que soluções de segurança para campus inteligente precisam enfrentar. Tais desafios são descritos a seguir.

Desafios gerenciais. O gerenciamento eficiente de tarefas por um órgão de segurança de um campus inteligente é essencial para garantir velocidade no atendimento e evitar desorganização durante situações de emergência como assaltos ou arrombamentos. Na UFRN, a delegação de tarefas aos vigilantes ocorria por meio de ligação telefônica ou comunicação via rádio. A coordenação de esforços através desses métodos podia levar a atrasos devido à natureza conversacional do processo, o que podia fazer a diferença em situações de emergência. Além disso, o operador que delega as tarefas não possuía um nível profundo de informações sobre o posicionamento em tempo real da segurança universitária, ou seja, o planejamento tático também sofria com a utilização de métodos inadequados para atendimento. Portanto, faz-se necessário um método eficaz para o despacho de vigilantes às ocorrências, provendo-os com informações relevantes que os auxiliem na preparação para o atendimento e resolução das ocorrências.

Desafios comunicacionais. A deficiência comunicacional no gerenciamento de ocorrências de segurança afeta o atendimento e descrição de ocorrências a serem tratadas e isso pode comprometer a efetividade das ações de tratamento. Esse tipo de deficiência era clara no âmbito da UFRN. A comunidade universitária possuía uma linha telefônica para registro de ocorrências e, assim como nos desafios gerenciais, isso podia acarretar em atrasos de atendimento que podem ter influência significativa (e negativa) em 
situações emergenciais. A inexistência de métodos de autenticação durante o registro de ocorrências deixava o sistema à mercê de usuários mal-intencionados, o que podia fazer com que recursos fossem desperdiçados em ações que eram consequência de informações não confiáveis.

Desafios informacionais. Os métodos tradicionais utilizados pelo DSP na UFRN não forneciam suporte para coleta, visualização ou análise de dados importantes sobre as ocorrências. Essa é uma abordagem que vai na direção contrária a como os dados devem ser manipulados em um contexto de uma cidade ou um campus inteligente. Mesmo quando coletados e armazenados, os dados ficavam contidos em planilhas ou arquivos de texto, o que impedia uma análise mais precisa das informações que eles podiam representar.

\section{O Sistema Integrado de Gerenciamento de Ocorrências (SIGOc)}

O Sistema Integrado de Gerenciamento de Ocorrências (SIGOc) é uma plataforma que foi desenvolvida com o objetivo de apoiar o gerenciamento de ocorrências de segurança e atividades de rondas de segurança em um campus universitário, além de prover à comunidade universitária um meio de comunicação direto com o órgão responsável pela segurança. No caso da UFRN, o SIGOc provê aos supervisores da DSP um sistema Web através do qual é possível ter uma visão geral das ocorrências de segurança reportada nos campi da universidade, bem como uma visão em tempo real da localização dos vigilantes em serviço e de quais ocorrências estão em atendimento e quais ainda não foram atendidas.

O SIGOc contempla ainda dois aplicativos para dispositivos móveis, um voltado para os vigilantes e outro para a comunidade universitária (docentes, discentes e servidores da universidade). $\mathrm{O}$ aplicativo Vigilante $U F R N$ provê à Central de Supervisão da DSP a localização dos vigilantes em tempo real, bem como provê aos vigilantes informações sobre ocorrências a eles atribuídas através do sistema Web de gerenciamento das ocorrências. Por sua vez, o aplicativo Campus Seguro permite que a comunidade universitária registre ocorrências de segurança nas dependências dos campi da universidade, permitindo também que tais usuários acompanhem em tempo real se suas ocorrências já tiveram atendimento iniciado pela Central de Supervisão da DSP. A Figura 1 mostra como esses elementos estão interligados.

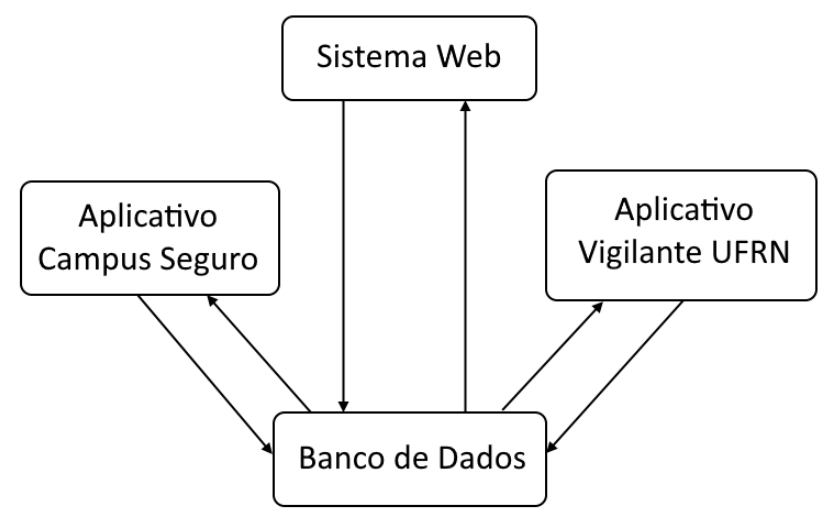

Figura 1. Arquitetura simplificada do SIGOc 
Ao utilizar o SIGOc, eventuais ocorrências serão registradas através do aplicativo Campus Seguro. Feito isso, operadores do DSP, utilizando o sistema Web, alocarão um ou mais vigilantes para atendimento da ocorrência. A equipe alocada receberá então uma notificação no aplicativo Vigilante UFRN e se dirigirão até o local indicado. Ao concluir o atendimento, a equipe de vigilantes finalizará o atendimento e a ocorrência é encerrada e armazenada para possíveis consultas. Após todo esse processo, fica disponível ao usuário a possibilidade de registrar seu feedback referente à ocorrência em questão.

\subsection{Aplicativos Campus Seguro e Vigilante UFRN}

O Campus Seguro é um aplicativo para dispositivos móveis voltados para discentes e servidores da UFRN e funciona como meio de comunicação e cadastro de ocorrências de segurança. Através do aplicativo, a comunidade universitária poderá cadastrar ocorrências intuitivamente, provendo informações valiosas ao aplicativo como categoria da ocorrência (assalto, furto de veículos, consumo de drogas, etc.), localização precisa da ocorrência acompanhada de pontos de referência e número de criminosos (ver Figura 2-a/b). Mesmo que a ocorrência tenha acontecido dentro de uma estrutura física em que a localização não possa ser obtida com precisão, o usuário pode mover o marcador que indica o local do ocorrido, adicionar novos pontos de referência ou inserir informações relevantes no campo de descrição da ocorrência. O usuário acessa as funcionalidades do aplicativo apenas após se autenticar através do sistema de autenticação integrada da UFRN, isto é, somente integrantes da comunidade universitária poderão usufruir do aplicativo. O Campus Seguro também conta com acompanhamento do status da ocorrência em tempo real e cadastro de feedback após a conclusão do atendimento.

O Campus Seguro contribui para aproximar a DSP e a comunidade universitária, facilitando a interação entre os dois ao oferecer um meio direto de comunicação entre ambos sem interrupções, além de prover meios de comunicação confiáveis, diretos e sujeitos a menos interferências. Para serviços de segurança, isso se faz extremamente necessário já que as informações coletadas podem determinar o sucesso das ações empenhadas. Para $\mathrm{o}$ atendimento emergencial de ocorrências, a velocidade e a praticidade presentes durante o preenchimento, envio e encaminhamento dos dados é imprescindível.

O cadastro e acompanhamento de ocorrências também são funcionalidades do aplicativo Vigilante UFRN, porém outros recursos estão presentes nesse aplicativo, tais como a visualização da ocorrência através de um mapa, finalização do atendimento de uma ocorrência e notificação de chegada ao local da ocorrência (ver Figura 2-c/d). Outra funcionalidade é o status do vigilante, que mostra ao operador do sistema Web se o vigilante está disponível ou não para alocação a uma ocorrência. Quando o status do vigilante é "Disponível", ele pode ser alocado, porém se o status for "Em Atendimento", isso significa que ele já está alocado em outra tarefa. Outros status possíveis são "Indisponível" e "Em Pausa", o que pode representar pausas para almoço, ida ao banheiro ou abastecimento de viatura. De maneira automática, ao se autenticar na aplicação, o vigilante passa a compartilhar sua localização com os operadores do sistema Web em intervalos de três segundos, o suficiente para limitar o uso de espaço e banda sem comprometer o acompanhamento realizado pelos supervisores. Em casos nos quais o sinal do GPS é ausente ou insuficiente, o sistema Web mantém a última posição registrada por aquele vigilante até que o aparelho do vigilante sinalize uma nova localização. 

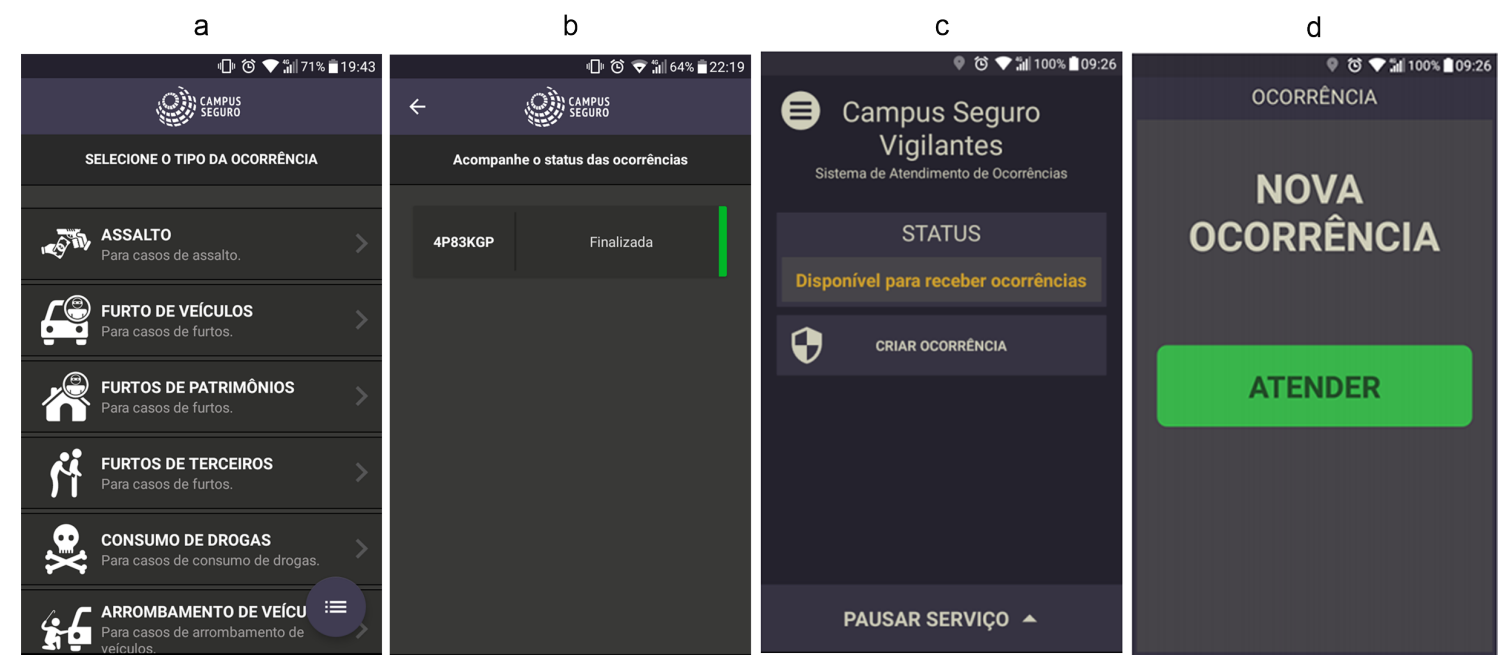

Figura 2. Aplicativos Campus Seguro (a/b) e Vigilante UFRN (c/d).

\subsection{Sistema Web}

O sistema Web da plataforma do Campus Seguro funciona como painel de controle para gerenciamento dos vigilantes e das ocorrências nos campi da UFRN (Figura 3). Esse sistema é utilizado por um operador para criar novas ocorrências e atribuir equipes de vigilantes a uma determinada ocorrência. Além disso, o operador pode adicionar, remover ou modificar vigilantes e ocorrências, bem como visualizar relatórios sobre os eventos no campus, como histórico de ocorrências.

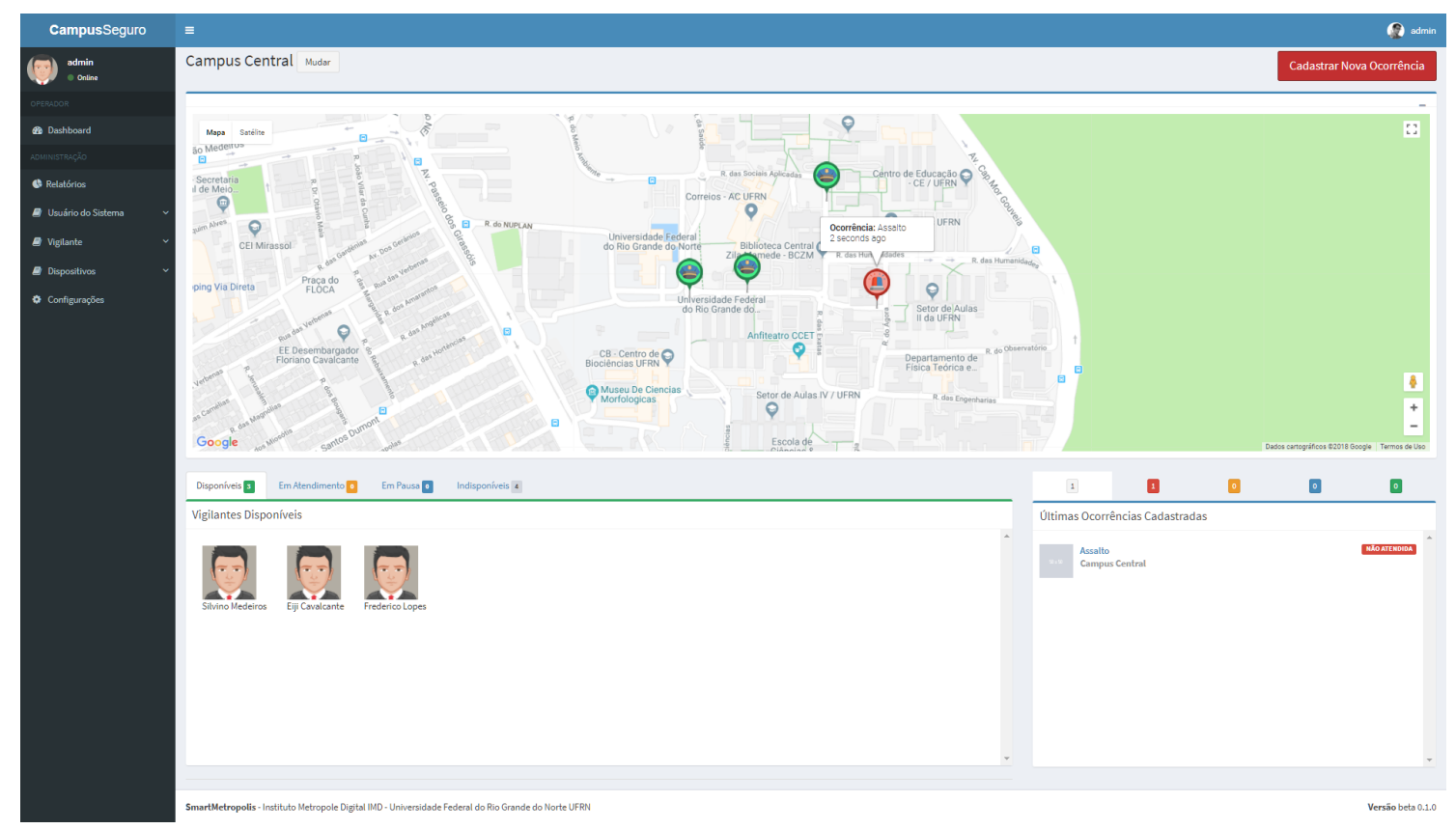

Figura 3. Tela inicial de acompanhamento do sistema Web

A adição do sistema Web à plataforma traz vários benefícios ao gerenciamento de segurança no campus. No momento do cadastro de uma ocorrência, o supervisor pode verificar dados importantes como usuário que a cadastrou, data e hora do cadas- 


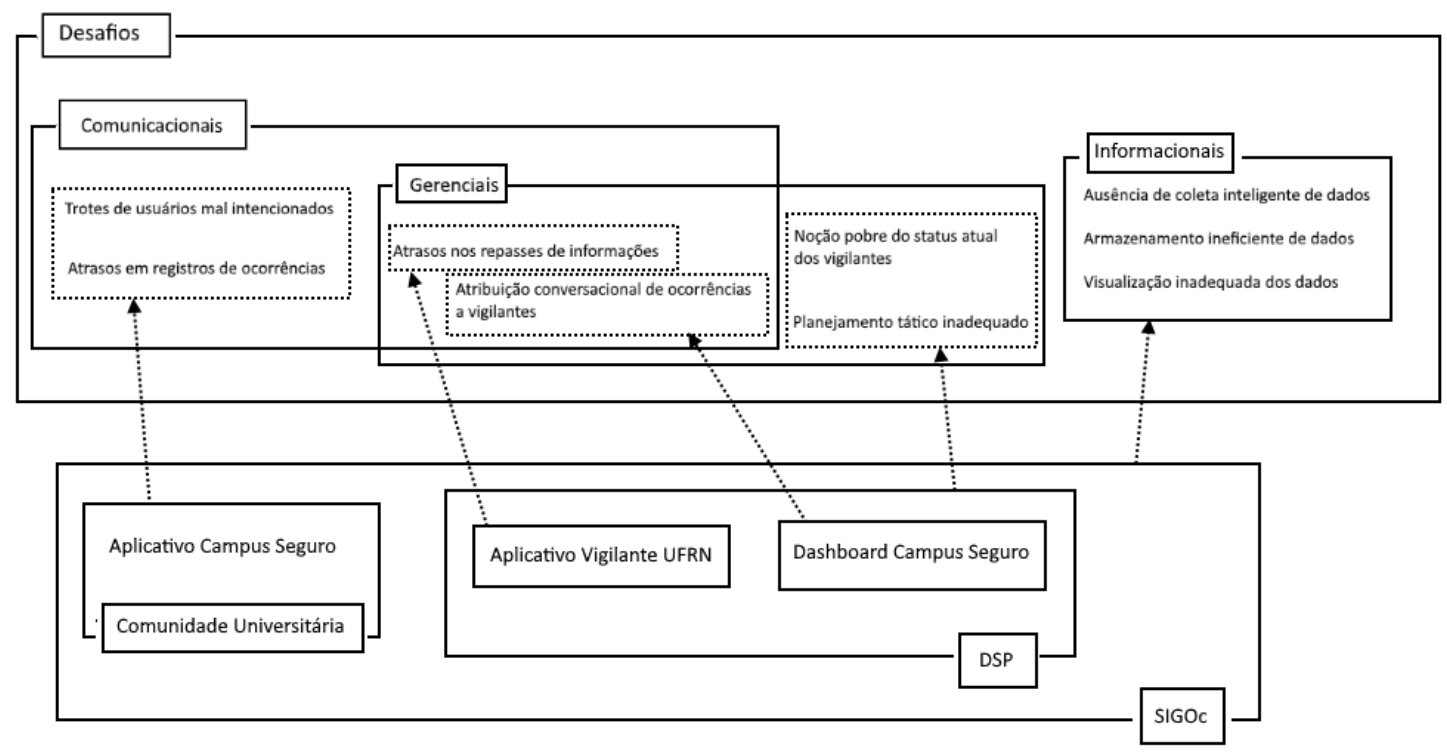

Figura 4. Relacionamento entre desafios encontrados e soluções propostas

tro, localização e tipo da ocorrência, e é o supervisor que fica responsável por atribuir qual vigilante deve atendê-la. Esses dados atualmente são repassados por telefone e o sistema Web padroniza essas informações, deixando clara ao operador a situação atual da ocorrência, reduzindo os riscos de falhas comunicacionais entre a comunidade universitária e a DSP.

Outro recurso importante provido pelo sistema Web é a visualização e gerenciamento de vigilantes em tempo real. Apesar de os vigilantes serem ainda despachados manualmente para o atendimento de ocorrências, essa atividade é agora feita com mais eficácia, já que o operador sabe o local exato da ocorrência e os vigilantes próximos a ela. De posse dessas informações, o operador seleciona a equipe de um ou mais vigilantes e os despacha para atender essa ocorrência. Esse alto nível de granularidade no processo tanto torna o gerenciamento de vigilantes e ocorrências mais robusto, com maior confiabilidade e precisão, quanto aumenta a qualidade das informações coletadas, agora mais precisas e confiáveis. As informações coletadas e armazenadas pelo sistema Web são posteriormente utilizadas para processamento e geração de relatórios.

\section{Análise da Adoção do SIGOc no âmbito da UFRN}

$\mathrm{Na}$ análise de como o SIGOc pode auxiliar a comunidade universitária e o DSP da UFRN nas questões relacionadas a ocorrências de segurança, foi possível observar como cada elemento da plataforma aborda um ou mais dos desafios identificados (ver Seção 2) e como a união dessas partes também colabora de um modo diferente para endereçá-los. O diagrama ilustrado na Figura 4 sintetiza essas relações entre os desafios e as soluções propostas.

O aplicativo Campus Seguro é direcionado à comunidade universitária e aborda principalmente o problema da comunicação desta com a DSP. O contexto comunicacional é melhorado substancialmente, visto que discentes e servidores agora possuem uma maneira rápida de reportar ocorrências e os operadores do sistema Web na DSP visualizam 
essas ocorrências com informações pertinentes, como uma maior precisão da localização, que muito provavelmente seria perdida ou de menor qualidade na comunicação oral. Os operadores do sistema Web, com base nessas informações, podem gerenciar os vigilantes com mais precisão, ou seja, o contexto gerencial também é melhorado.

Ainda no contexto gerencial, é possível perceber que o aplicativo Vigilante UFRN, junto do sistema Web, traz recursos que aprimoram os processos de gerenciamento da DSP. O aplicativo repassa informações primordiais ao gerenciamento de vigilantes, como a localização atual do vigilante, status de atendimento de uma ocorrência e status atual do vigilante, informações essas que são mostradas no sistema Web e são essenciais para alocação dos vigilantes no atendimento de uma eventual ocorrência. Além disso, os recursos do sistema Web fornecem um controle muito mais refinado sobre o gerenciamento de vigilantes e ocorrências, o que seria mais difícil de ser alcançado apenas através de rádio e ligações telefônicas.

No contexto informacional, o SIGOc como um todo propicia maneiras sofisticadas de armazenamento e processamento utilizando os dados obtidos a partir dos elementos que fazem parte da plataforma. Por exemplo, as localizações dos vigilantes são armazenadas no banco de dados da plataforma e diversas análises podem ser realizadas sobre desses dados, revelando padrões de deslocamento e distribuição espacial dos vigilantes. O sistema Web também possui recursos de gerenciamento, permitindo cadastro, atualização e remoção de usuários e vigilantes.

\section{Trabalhos em Andamento e Futuros}

As deficiências comunicacionais, informacionais e gerenciais que se fazem presentes no contexto atual de gerenciamento de ocorrências nos campi da UFRN foram as principais motivações para o desenvolvimento do SIGOc e as soluções associadas. Apesar de já contar com recursos que otimizam o atendimento de ocorrências e o gerenciamento de vigilantes, o SIGOc ainda contém recursos importantes a serem implementados. Por exemplo, ocorrências podem ser atribuídas apenas a vigilantes com status "Disponível", ou seja, vigilantes com status "Em Atendimento" não serão considerados para atribuição, impedindo realocação de pessoal em atendimento mediante à ocorrências concorrentes.

Atualmente, as aplicações que constituem o SIGOc estão em operação no campus central da UFRN, na cidade do Natal, e já são capazes de abordar os desafios anteriormente mencionados para contribuir com a melhoria da segurança por meio da gestão eficiente de ocorrências. Todavia, alguns recursos passaram a se fazer necessários para melhorar ainda mais esses fatores, os quais são discutidos a seguir.

Implementação das Vias Azuis. A dimensão geográfica da UFRN traz dificuldades com relação à cobertura da segurança. A vigilância não consegue monitorar toda a região e, por isso, alguns locais estão mais propensos a ocorrências, locais estes muitas vezes percorridos pela comunidade universitária. As vias azuis serão trajetórias distribuídas pela Universidade onde a vigilância é fortalecida através de câmeras e vigilantes. O aplicativo Campus Seguro proverá funcionalidade para calcular as Vias Azuis, ou seja, trajetórias dentro dos campi da UFRN com maior cobertura de câmeras de segurança e pontos de ronda dos vigilantes para que alunos e servidores se desloquem com maior sensação de segurança dentro da UFRN;

Botão de Pânico. O Botão de Pânico funcionará como ferramenta para reportar 
rapidamente alguma ocorrência urgente dentro do campus, o que pode ser uma ferramenta útil principalmente em uma situação urgente ou de tensão do usuário, como no caso de um assalto. Atualmente, o aplicativo Campus Seguro requer vários passos a serem seguidos antes da ocorrência ser registrada, o que pode dificultar a sua utilização em casos emergenciais, nas quais o usuário precisa cadastrar a ocorrência rapidamente.

Relatórios. O sistema Web, em sua versão atual, é capaz de gerar relatórios para possibilitar consultas de dados sobre ocorrências e vigilantes. Contudo, o formato de apresentação desses dados é muito pobre e mostra apenas informações básicas sobre o tópico do relatório. No momento, não é possível correlacionar informações e obter um relatório de uma lista das ocorrências cadastradas em um intervalo de tempo. Portanto, esse refinamento é necessário para que o processamento dos dados armazenados pelo sistema resulte em informações de maior valor agregado que permitam análises mais significativas através do sistema.

\section{Conclusão}

Cidades inteligentes possuem necessidades variadas que vão desde o engajamento cívico até processamento e armazenamento de dados de forma adequada e que gere informações de valor agregado para apoiar processos de tomada de decisão por parte dos usuários. Alguns desafios decorrentes da deficiência em atender essas necessidades podem se manifestar tanto na própria cidade inteligente quanto em um campus universitário. Através da observação desses desafios no âmbito do gerenciamento de segurança na UFRN, este trabalho propôs a plataforma SIGOc como uma solução composta de dois aplicativos para dispositivos móveis e um sistema Web como um painel de controle no intuito de contribuir para melhoria da qualidade dos processos de gerenciamento de ocorrências pela DSP. Através de uma maior compreensão da problemática e o uso já em andamento da solução, trabalhos futuros podem ser realizados visando um aumento da segurança da comunidade universitária e melhora ainda mais sensível dos processos gerenciais da DSP.

\section{Referências}

Barrionuevo, J. M., Berrone, P., Ricart, J. E. (2012) "Smart cities, sustainable progress", IESE Insight 14, pp. 50-57.

Ferreira, J. E., Visintin, J. A., Okamoto Jr., J., Pu, C. (2017) "Smart services: A case study on smarter public safety by a mobile app for University of São Paulo", Proceedings of the 2017 IEEE Conference on Smart City Innovations. USA: IEEE.

Lacinák, M., Ristvej, J. (2017) "Smart city, safety and security”, Procedia Engineering 192, pp. 522-527. 